\title{
Lantibiotics: A Candidate for Future Generation of Antibiotics
}

\author{
S. M. Asaduzzaman, ${ }^{1 \ddagger}$ Abdullah-Al-Mahin, ${ }^{1}$ Tanvir Bashar, ${ }^{2}$ and Rashed Noor $^{2}$ \\ ${ }^{1}$ Institute of Food and Radiation Biology, Atomic Energy Research Establishment; ${ }^{2}$ Department of \\ Microbiology, Stamford University Bangladesh
}

Received 9 September 2010/Accepted 5 October 2010

\begin{abstract}
Emergence of multi-resistant pathogens has urged the exploration of antibiotic repertoires. The long reliability on the glycopeptide antibiotic vancomycin against fatal diseases caused by Gram-positive bacteria is also in wrecked to insist on finding more promising alternative antibiotics. The peptide antibiotic nisin is effective against vancomycin resistant bacteria. Nisin is the first lantibiotic that was discovered before penicillin and has been widely used as a safe alternative for chemical reagents in food preservation in around 50 countries for over 40 years without natural resistance development. Therefore, lantibiotics are one of the most promising alternative candidate for future antibiotics that maintain their antibacterial efficacy by many modes of action. Till now, more than 60 lantibiotics have been isolated, identified and characterized. We explained various aspects of biosynthesis, grouping, mutagenesis, activities and modes of action of lantibiotics in this review.
\end{abstract}

Identification of penicillin in 1928 was a historical milestone. Subsequent discovery and production of a wide variety of antibiotics on a massive scale has revolutionized approaches to human civilization. Healing of individuals with otherwise incapacitating and sometimes fatal infectious diseases by antibiotics has been considered as naught short of medical miracle.

Unfortunately, shortly after the initial wide-spread use of antibiotics has generated a strong evolutionary pressure for the emergence of bacteria that either have an inherent resistance to a particular antibiotic or have the capacity to acquire such resistance. Rapid emergence of multi-resistant pathogens has made the problem further complex for an almost exclusive reliance on broad-spectrum antibiotics. These devastating threats of acquiring nuisance resistance to antibiotics are compounding from all corners of antibiotics' end-users, e.g, agricultural and food production settings, disease control and prevention of animal and plant kingdoms.

Of which, dangerous percentage of resistance are acquired from hospital settings, where an intensive use of antibiotics is sometimes unnecessary, poorly chosen or incorrectly administered. Consequently, today there is no antibiotic in clinical use to which resistance has not developed, and so there is a pressing need to discover and/or develop new agents that are active even against resistant bacteria.

${ }^{\ddagger}$ Corresponding Author. Mailing address: S. M. Asaduzzaman, Institute of Food and Radiation Biology, Atomic Energy Research Establishment, Savar, Dhaka, Bangladesh. Phone:+88-02-7789228, Fax: +88-02-8130102, Email: sikderasad@yahoo.com.
In search of alternative antibiotics, some antimicrobial agents are now being considered, such as bacteriocins, bacteriophage, probiotics and antimicrobial peptides. Finding of new classes of antibacterial compounds based on targets identified from bacterial genomics has led to renewed interest in natural products, which have historically been invaluable as a source of antibacterial drugs, such as glycopeptides reflecting their evolutionary origin as 'weapons' that bacteria use against each other. One such glycopeptide antibiotic, vancomycin had long been reliable in treating infections caused by bacteria resistant to several other antibiotics and is usually reserved for treatment of serious infections, including those caused by the 'super bug' methicillin-resistant Staphylococcus aureus (MRSA). But even vancomycin resistance has now become quite common (1) underlining the necessity for new and more effective antibiotics. World Health Organization has warned that the vivid increase of resistance among pathogens may become untreatable (2). Therefore, these dramatic rises in antibiotic-resistant pathogens have stimulated renewed efforts to identify, develop or design antibiotics that may be active against the multiresistant pathogens.

\section{LANTIBIOTICS}

Surprisingly, the history of lantibiotics is older than that of conventional antibiotics, and dates back before discovery of penicillin. The first lantibiotic nisin was discovered in 1920s that has widespread application as a safe alternative for chemical reagents in food preservation in around 50 countries for over 40 years without natural resistance development $(3,4)$. 
Research on lantibiotics has recently gained renewed interest due to the emergence of clinical isolates resistant to antibiotics like vancomycin, the drug of last resort against infections caused by Grampositive bacteria for almost thirty years. $N$-acyl-DAla-D-Ala moiety of lipid II is involved for binding of vancomycin, and vancomycin resistant bacteria remain sensitive to nisin (5).

Therefore, there is a spurred and rapid expansion of research activities towards lantibiotics throughout the world for last few years. Despite the oldest known antibacterial agent, the structure of nisin was not determined until the elegant landmark studies by Gross and Morell in 1971 (6), and the word "lantibiotic" was just coined in 1988 as an abbreviation for lanthionine-containing antibiotic peptides (7) after getting the renewal interest from the thinking on it to be the most possible and plausible alternative candidates of antibiotics. Therefore, though the history of lantibiotics is very old but now it is thinking for a new paradigm due to its hopeful and enormous chemotherapeutic potential to peep up the present need, and to meet the challenges of the future demand for developing superior antibiotics to combat even against the emerging pathogens.

Bacteria are remarkable producers of antimicrobial peptides. In the past decades, there were massive reports of such antibacterial compounds in the scientific literatures. Bacteria-derived antimicrobial peptides have a large degree of structural and chemical diversity. Polypeptide antibiotics (e.g., gramicidin and valinomycin) are synthesized by large, multi-enzyme complexes from building blocks provided by a variety of cellular processes (8).

Recent advances on bacterial molecular genetics have further contributed to find new insight of peptide antibiotics. Ribosomally synthesized peptide antibiotics produced by certain bacteria are termed as bacteriocins $(9,10)$. Bacteriocins are divided into some classes, among them lantibiotics are class-I bacteriocins that are antimicrobial peptides containing unusual amino acids, such as thioether-cross linked amino acid, lanthionine and 3-methyllanthionine, and dehydrated amino acids, e.g., 2,3-didehydroalanine (Dha) and (Z)-2,3-didehydrobutyrine (Dhb) (11, 12). Post-translational modification renders the lantibiotics to yield their biologically active forms. A large variety of lantibiotics have currently been reported and their structures, biosynthetic mechanisms, and mode of actions have drawn significant research interests.

\section{UNIQUE FEATURES OF LANTIBIOTICS}

Lantibiotics display a number of notable characteristics. These are ribosomally synthesized and the genes involved in lantibiotic biosynthesis are clustered in most cases that are designated by the generic locus symbol lan, with a more specific genotypic designation for each lantibiotic member (e.g., nis for nisin, nuk for nukacin ISK-1, gdm for gallidermin). They are found on conjugative transposable elements (e.g., nisin), on the chromosome of the host (e.g., subtilin), or on plasmids (e.g., lacticin 481). The gene clusters for the biosynthesis of representative lantibiotics are depicted in Fig. 1. Although the gene order, complexity, and transcriptional organization of the various clusters differ, three genes (lanAMT) have been identified that are involved in the biosynthesis of all type-A(II) and type B lantibiotics, and four genes (lanABCT) are present in all type-A(I) lantibiotics gene clusters. These essential genes obviously include the structural genes encoding the precursor peptides for posttranslational maturation (prepeptides), which have been designated lanA, except for subtilin whose structural gene historically is named spaS. The lanA genes produce prepeptides that have an extension (leader peptide) of 23-59 amino acids at their $\mathrm{N}$ terminus in addition to the mature lantibiotic. Sequencing of the $\operatorname{lan} A$ genes indicated that Ser and Thr residues are the precursors of Dha and Dhb structures found in the final products, and that Ser plus Cys and Thr plus Cys residues are the precursors to form the characteristic lanthionine and methyllanthionine structures, respectively. Though the exact function of leader is not clear yet, the possible functions that have been suggested include signaling for export, protection of the producing strain by keeping the peptides inactive and providing scaffolds for the post-translational modification machineries $(13,14)$.

In type-A(II) lantibiotics, the bifunctional LanM is responsible for dehydration and the cyclization reactions, in contrast to type-A(I) lantibiotics in which two genes, lanB is involved in dehydration of Ser and Thr to form Dha and Dhb, respectively, and lanC codes for cyclase that produces lanthionine or methyllanthionine (Fig. 2).

The C-terminus of lanM enzyme contains $20-27 \%$ sequence identity to the lanC enzyme, but, it has no homology to lanB enzyme. Recently, the direct evidence for the bifunctional role of lanM enzyme for catalyzing dehydration and cyclization has been shown by in vitro reconstitution of lctM in lacticin 481 biosynthesis (15). In addition to the characteristics dehydration and lanthionine bridge formation, some lantibiotics undergo further post-translational modifications. For example, the unusual amino acids $S$-[(Z)-2-aminovinyl]-D-cysteine (AviCys) and $S$-[(Z)2-aminovinyl]-(3S)-3-methyl-D-cysteine (AviMeCys) are formed at the C-terminus of epidermin and mersacidin, respectively. The $\operatorname{lan} D$ genes encode the enzyme responsible for the formation of AviCys and AviMeCys, likely the epiD in epidermin and mesD in mersacidin carry out the in vitro decarboxylation of a C-terminal Cys residue to form AviCys and AviMeCys, respectively $(16,17)$. 


\section{Nisin}

Type-A(I)

Nukacin ISK-1

Type-A(II)

\section{Mersacidin}

(Type B)
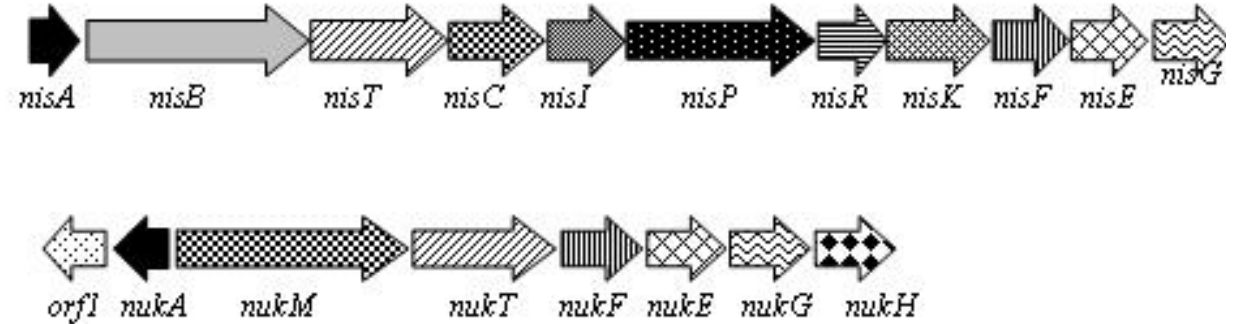

orfl $n u k A \quad n u k M$

FIG. 1. Representative biosynthetic gene clusters of lantibiotics. Functional similarity of the proposed genes have been highlighted by the same colors/wedges.

NisA Leader- ITSISLCTPGCKTGALMGCNMKTATCHCSHHVSK

$$
\text { NisB (dehydratase) }
$$

Leader- IDhbDhalDhaLCDhbPGCKDhbGALMGCNMKDhbA DhbCHCSIHVDhaK
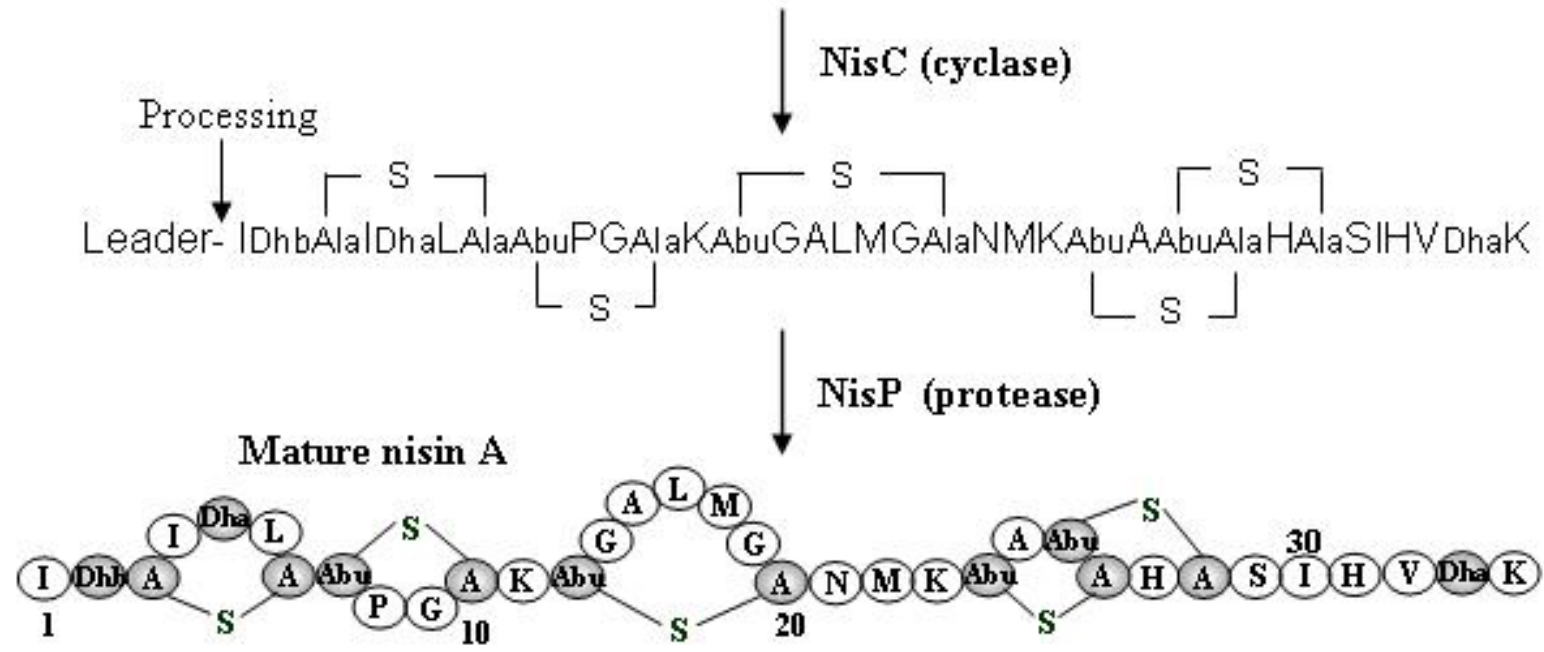

FIG. 2. Representative example of the post-translational maturation process of lantibiotic nisin A. Serine and threonine residues (bold) in nisin prepeptide are dehydrated by NisB. Cyclization of dehydrated amino acids with cysteine residues are catalyzed by NisC in a regio- and stereo-specific manner and the protease NisP then cleaves proteolytically of the leader peptide. 
The N-terminal leader peptide is cleaved off and the mature lantibiotic is then translocated across the membrane. The prepeptide of type-A (I) lantibiotics is translocated via ATP binding cassette transporter LanT, and the leader peptide is catalyzed by serine protease LanP. However, recent report on broad substrate specificity of NisT for nisin biosynthesis suggested secretion of unmodified, dehydrated, cyclized nisA prepeptide and non-lantibiotic peptide fused to the leader peptide of nisA (18). In contrast to the broad specificity of NisT, the processing enzyme NisP only removes the leader peptide attached to fully modified nisin. In type-A(II) lantibiotics, LanT has dual functions, which removes the leader peptide and exports the matured peptide. It has an extra N-terminal cysteine peptidase domain compared to LanT of typeA(I) lantibiotics (19).

Some gene clusters contain a second transport system that usually consists of three genes (lanEFG) and is concerned with the immunity of the producerstrains. Besides, another gene designated by lanI is also assumed to be concerned with self-immunity to some lantibiotics (20). Additionally, two directive genes (lanKR) are often found for regulation of lantibiotic biosynthesis encompassing an important two-component sensory system (21).

\section{DIVERSIFIED STRUCTURES AND LANTIBIOTICS' GROUPING}

Presently, as many as 50 different lantibiotics have been isolated from Gram-positive bacteria. Lantibiotics are classified by Jung (22) as type A and $\mathrm{B}$ based on the topology of their structures. Representatives of the structures of lantibiotics have been presented in Fig. 3. Type A lantibiotics are further divided into two subtypes, elongated type-A(I), and tail and ring region containing type-A(II), which have their different genetic organizations (23). In type-A(I) lantibiotics, the lanthionine and 3methyllanthionine residues are formed by the action of two distinct enzymes (lanB and lanC), whereas those that are formed by a single enzyme (lanM) are termed type-A(II) (24). Type B lantibiotics, such as mersacidin, cinnamycin, duramycin B and $\mathrm{C}$, and ancovenin are more globular and compact in structure.

Besides, a separate subgroup is formed by the twocomponent lantibiotics consisting of two posttranslationally modified peptides that individually have weak or no activity but synergistically display strong antibacterial action. At present, this emerging subgroup of two-component lantibiotics encompasses structurally closely related lacticin 3147 , plantaricin W, staphylococcin C55 and the completely unrelated streptococcal cytolysin, which combines bacteriocin and cytolytic activity against blood cells (25).
Molecular concepts for the synergistic effect of two bacteriocin peptides are not clearly available at present. Generally the two-peptide lantibiotics work best at equimolar concentrations (1:1 stoichiometry). However, an alternative classification of lantibiotics (24) has been proposed by comparing the leader sequences of many lantibiotics that reveal two different conserved motifs other than those presented above [i.e., type-A(I), type-A(II), and type B)]. lantibiotics that reveal two different conserved motifs other than those presented above [i.e., type-A(I), type$\mathrm{A}(\mathrm{II})$, and type $\mathrm{B}$ )]. In this organization (by genetics rather than activity profiles or three-dimensional structure), the class I lantibiotics all have a common "FNLD" motif between positions -20 and -15 and usually a Pro at position -2. The biosynthetic machinery involved in the post-translational modifications in this class consists of LanB and LanC. In contrast, class II peptides contain a characteristic "GG" or "GA" cleavage site (historically termed the "double Gly motif"), contain multiple Asp and Glu residues, and are usually processed by one modification enzyme (LanM).

\section{STRUCTURE-FUNCTION RELATIONSHIPS OF LANTIBIOTICS}

Structural-functional relationships of lantibiotics are quite diverse. Natural variants of lantibiotics suggest that the presence of certain amino acids in specific locations are crucial, whereas the other amino acids are flexible (redundant) and thus be possible to generate mutants that enhance a particular phenotypic trait of the peptide without reducing activity. Both the conserved and flexible regions may demonstrate a greater propensity to change and therefore can permit the comparison with the other peptides most likely to be essential for activity. Among the numerous natural variants of lantibiotics, the most common to cite for examples are the three variants of nisin [a type-A(I) lantibiotic]. Nisin A differs from nisin $\mathrm{Z}$ by having a histidine rather than an asparagine residue at position 27 (26). Nisin Q differs from nisin A in a greater way having Met17Glu, Met21Leu, His27Asn, Ileu30Val (27). The activity-spectra of the peptide variants are similar (28). Another example of the existence of two very closely related natural variants of lantibiotics is gallidermin, which differs from epidermin by a leucine rather than an isoleucine at position 6 and gallidermin has relatively higher antibacterial activity against pathogenic Gram positive bacteria (e.g., S. aureus SG 511 and Propinib. acnes IF 31002) (29).

Type-A(II) lantibiotics have uniform structures with the same ring patterns containing four unusual amino acids, but have not yet been studied well to distinguish their activities that result from structural-positional differences for specific positions. 


\section{Type-A(T)}

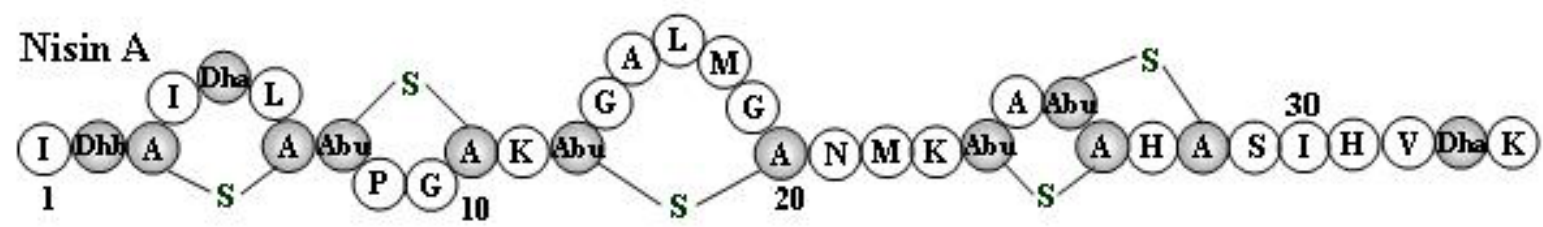

Subtilin

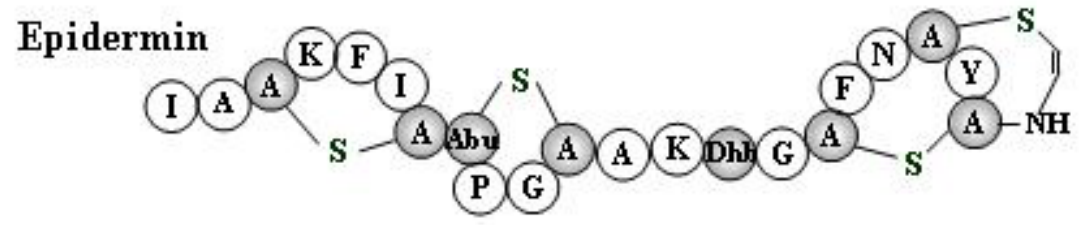

Type-A(I)

Nukacin ISK-1

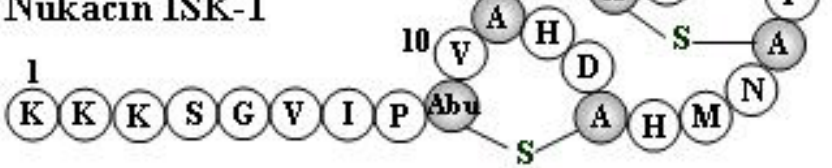

\section{Lacticin 481}<smiles>c1ccc(-c2ccccc2)cc1</smiles>

FIG. 3. Major types of lantibiotics showing their representatives structures. The dark-shaded residues indicate modified amino acids. AS-A, lanthionine; Abu-S-A, 3-methyllanthionine; Dha, dehydroalanine; Dhb, dehydrobutyrine; A-S-CH=CH-NH-, S-[(Z)-2-aminovinyl]D-cysteine; Abu-S-CH=CH-NH-, S-[(Z)-2-aminovinyl]-(3S)-3-methyl-D-cysteine; D-A, D-alanine. 
Dufour et al. (30) have recently reviewed about the type-A(II) lantibiotics that include more than sixteen lantibiotics and are mentioned as the largest among the groups of lantibiotics, but these are the simplest in both structurally and biosynthetically compared to other groups of lantibiotics. Structurealfunctional relationships of lantibiotics have been discussed below focusing on engineering that include the activities of structural variants of lantibiotics, and about the involvement of structures governing mode of actions.

\section{ENGINEERING OF LANTIBIOTICS}

One of the unique features of lantibiotics is that they are gene-encoded and therefore can be subjected for mutagenesis. Mutagenesis of the lantibiotic structural genes has shown the feasibility of changing the structure of lantibiotic by genetic engineering. Till now, nisin is the most widely engineered structure that has been extensively investigated for structurefunction relationships. However, heterologous expression systems have also been established for many other lantibiotics and are working in many laboratories on determining structural determinants for various lantibiotics throughout the world. Replacement of Dha by Dhb and vice versa has been reported for many lantibiotics. Formation of Dhb instead of Dha in the structural region at position 5 of the nis $Z$ gene led to the production of mature nisin $\mathrm{Z}$ (31) that shows an antibacterial activity by a 2-10-fold lower against many indicator strains (32). Dhb10Dha mutant of mutacin II was reported to have similar activity (33), whereas the mutant Dhb14Dha did not show any noticeable change of activity of gallidermin (32). Introduction of a new thioether bridge in the lantibiotic Pep5 resulted in dramatic decrease of antimicrobial activity (34).

Usually, replacement of dehydrated amino acid residues in lantibiotics by other amino acids reduces their antibacterial activity. The mutant Dha5Ala has almost the same activity of wild-type nisin against vegetative cells but nearly abolishes the activity against spores (35). Removal of Dha33 by Ala or change of Dha5 and Dha33 with Ala lead to remarkable decrease of activity, which is about $1 \%$ activity of wild-type nisin (36).

Replacements of Dhb by Ala at positions 16 and 20 of Pep5 are found to lower the activity toward some indicator strains (4). The mutant Dha16Ile in mersacidin causes great reduction of its activity against $M$. luteus and $S$. pyogenes (37). Since the replacements of amino acids in all positions are not tolerated by the biosynthetic machineries, so are not expressed.
For instance, an attempt to generate the mutant Dhb10Ala mutacin II leads no detectable mutacin production (33), and change of Ser3, Ser19, or Cys22 that forms the lanthionines also resulted in loss of production of gallidermin (38).

Introduction of negatively charged amino acids in the C-terminal part of nisin $\mathrm{Z}$ causes significant reduction of activity (39), whereas introduction of positively charged lysine residues in the hinge region of nisin $\mathrm{Z}$ significantly changes its properties with increased conformational flexibility, which augments antimicrobial activity to kill Gram-negative bacteria (40). Rollema et al. (41) reported that the presence of lysine residues in nisin molecule considerably enhances its solubility at neutral $\mathrm{pH}$.

\section{MODE OF ACTIONS OF LANTIBIOTICS}

The activities of lantibiotics are mostly based on different killing mechanisms that are combined in one molecule. For example, the prototypic lantibiotic nisin inhibits peptidoglycan synthesis and forms pores through specific interaction with the cell wall precursor lipid II (42). Another example of a different scenario is that the mutant [A12L]gallidermin, in which the ability to form pore is disrupted but is as potent as wild-type gallidermin, indicating that pore formation does not contribute to killing. In contrast, nisin rapidly forms pores in the $L$. lactis subsp. cremoris HP but it is about 10-fold less efficient in killing (43). It has now become well known that the multiple activities of lantibiotics combine differently for individual target strains. However, the general pictures of the lantibiotics activities may be attained by, i) binding to bacterial membrane and then insertion into membrane, and ii) use of receptor/docking molecules to exert structure-based activity.

\section{BINDING OF LANTIBIOTICS TO MEMBRANE AND INSERTION INTO MEMBRANE}

Many studies showed that membrane binding is the first among the steps of lantibiotics mode of actions. Altering the charge distributions in nisin, for example, removal of positive charge from the $\mathrm{N}$ - or C-termianl region of nisin hampered the initial interactions of peptide and membrane (44). Comparing native nisin with its variants, they also reported that electrostatic attractions encourage the initial association of nisin with membrane. Negative charge into nisin $\mathrm{Z}$ resulting in electrostatic repulsion with the negatively charged phospholipids. 
Strongly reduced binding affinity of nisin (1-12) fragment for anionic phospholipids further indicated the importance of the C-terminus for binding to the membrane $(45,46)$. However, though the initial binding to the membrane surface seems to involve the C-terminus of nisin, but studies with a variant of nisin $\mathrm{Z}$ in which a short peptide has been fused into its Cterminus shows that the C-terminus translocates across the membrane. This translocation of the C-terminus is correlated with pore-forming activity and both the activities are anionic lipid dependent (47). Once bound electrostatically, the peptide adopts a membrane spanning orientation in which at least part of the molecules that form the pore has their C-terminus in the lumen of the vesicle. However, it is now clear that the N-terminal rings of nisin bind to the disaccharidepyrophosphate region of lipid II in which the positively charged C-terminus initially interacts with the head-groups of the lipids in the membrane bilayer. Demel et al. (48) reported that lacticin 481 has higher affinity for zwitterionic membrane in contrast to nisin that binds to anionic membrane.

\section{PORE FORMATION}

Nisin and many other cationic type-A(I) lantibiotics are subjected to study the mode of actions using cytoplasmic and artificial membranes (11). Numerous studies prior to the late 1990s focused on the permeabilization of bacterial cell membranes as the primary mode of action of nisin and other type-A(I) lantibiotics. The lifetimes of pores may be a few to several hundred milliseconds and with diameters up to $2 \mathrm{~nm}$ (49). Pores of nisin are somewhat anion selective (45) and the pores of nisin and Pep5 work only in one direction (rectifying) (50, 51), whereas gallidermin and epidermin form nonrectifying channels that are more stable (52). The model membrane systems, such as planar lipid bilayers and liposomes have strong influence on the efficiency of pore formation (53). It has also been shown in monolayer study that antimicrobial activity is correlated well with the nisinanionic lipid interaction $(48,53)$. The two most well established mechanisms of pore formation are the barrel-stave and the wedge model. In barrel-stave mechanism, the cationic lantibiotic monomers bind to the membrane-surface through electrostatic interaction and are assembled into a pre-aggregate, and pores are formed at a certain membrane potential where the lantibiotic gets a position perpendicular to the membrane (49). In case of wedge model, surfacebound lantibiotic molecules bind parallel to the membrane surface and generate local strain leading to bending of the membrane in a such way that the lipid molecules together with lantibiotic make up the pore (54).
Chikindas et al. (55) proposed a model for the orientation of lantibiotic in negatively charged membranes in which the relatively elongated nisin molecule lies parallel to the membrane surface with the positively charged side-chains of amino acids, pointing out of the lipid bilayer. In contrast to this model that stands for the most stable orientation, transient pore formation may use the molecule to pass through the membrane by conformational change. Two attractive models for pore formation by lantibiotics have been presented in Fig. 4.Pore formation by nisin is unique compared to vancomycin, teicoplanin, and ramoplanin in that it subsequently binds with lipid II using as a docking molecule to form pore that is stable and highly efficient (56). Fig. 4 (A) depicts the structure of lipid II that portrays the regions involved in binding of different antibiotics. Breukink et al. (5) reported that the presence of lipid II in membrane increases the pore-forming efficiency of nisin 1000-fold than the peptide that does not use lipid II. The efficiency of pore formation in membranes consisting of anionic phospholipids shown in the absence of lipid II is completely undone in its presence and the anion selectivity observed in model membrane systems are disappears upon addition of lipid II (42). Dramatic increase of nisin's activity is shown by lipid II-mediated pore formation in which only two lipid II molecules per $10^{5}$ phospholipid molecules greatly enhances the release of dyes from vesicles (5). Lipid II changes the orientation of nisin from parallel to perpendicular with respect to the membrane surface (57), and is recruited into a stable pore structure (56). Nisin binds to the disaccharidepyrophosphate moiety of lipid II through the $\mathrm{N}$ terminal rings, and the positively charged C-terminus initially interacts with the head groups of the phospholipids of membrane [Fig. 4 (A and B)]. The involvements of manifold molecules of the lipid IInisin complex (56) are subsequently comprehensive to form a defined pore of uniform structure. Therefore, the lipid II-mediated pore-complex is highly stable and is unique, because other membrane active cationic pore forming antimicrobial peptides form unstable transient pores and are not uniform in structures (58). Two component lantibiotic, lacticin 3147 has also been shown to utilize lipid II in a sequential manner to form a defined pore (Fig. 4D). However, as thought before that pore formation is the major killing mechanism of membrane active peptides, the idea has now been renewed and replaced with many novel works, which uncovered the structure-based activities of lantibiotic that are explained below. 
(B)
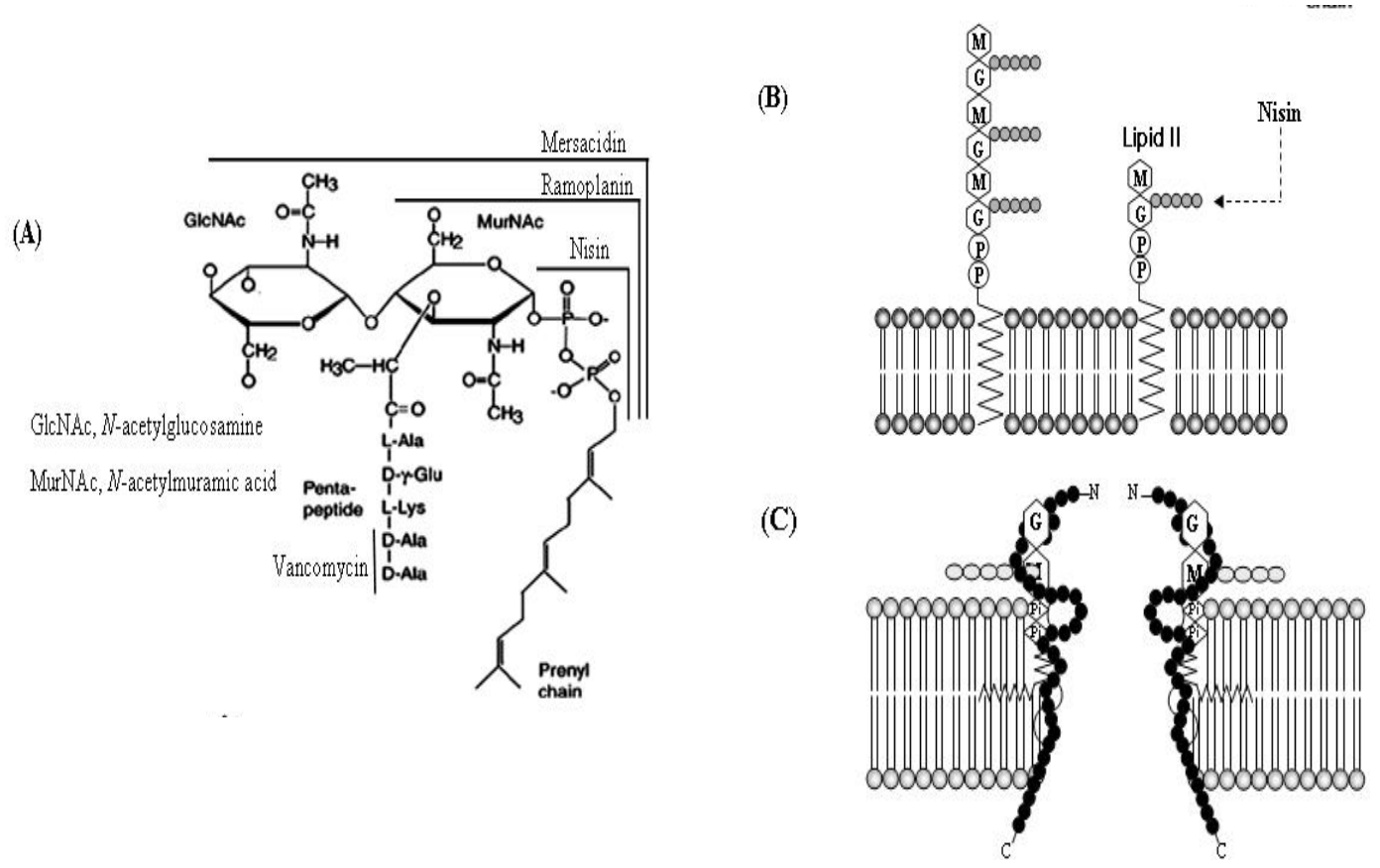

FIG. 4. Structure of lipid II and pore formation by lantibiotics. (A) Chemical structure of lipid II. NMR analysis of lipid II-nisin complex reveals that the $\mathrm{N}$-terminal part of nisin (residues 1-12) encages the pyrophosphate moiety of lipid II with hydrogen bonds network (38). (B) Nisin binds to cell-wall precursor lipid II using a docking molecule. (C) The N-terminus of nisin binds lipid II while the C-terminus inserts into bacterial membrane, subsequently makes a pore.

(D)

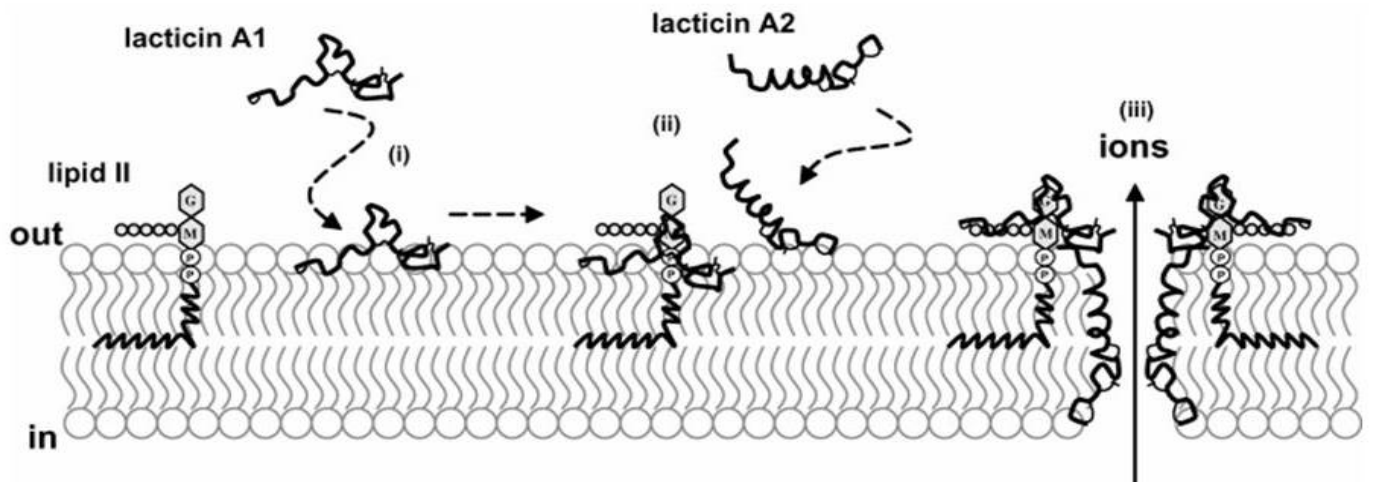

FIG. 1-4 (continued). (D) Pore formation by two component lantibiotic. Wiedemann et al. (87) proposed a three-step model for the antibiotic activity of the two component lantibiotic, lacticin 3147. The A1 component of lacticin associates with the membrane to bind lipid II in the first step (i). The A1 peptide bound with lipid II induces or stabilizes to facilitate the interaction with the A2 peptide and enables the formation of a two peptides-lipid II complex in step two (ii). Once bound, the A2 peptide is able to adopt transmembrane orientation enabling to form a defined pore (iii). 


\section{LIPID II TARGETING LANTIBIOTICS ACTIVITIES}

Bacteria-specific cell-wall precursors, e.g., lipid I and lipid II are essential for bacterial cell-wall biosynthesis. Many antibiotics bind to these precursors to interfere with peptidoglycan biosynthesis by physically sequestering them preventing utilization by transpeptidase and transglycosylase enzymes that install the cross-linked network of the bacterial cell wall. Vancomycin (a peptide antibiotic) is the example of first compound that kills bacteria by targeting lipid II and has long been reliable as an essential antibiotic. Vancomycin binds to the D-Ala-D-Ala moiety of lipid II and nisin binds to the disaccharides-pyrophosphate region of lipid II, so nisin is even effective against vancomycinresistant strains though both are confined to target lipid II (59). It has recently been demonstrated that lipid II is the prime target of several other classes of natural products including the lantibiotics. A growing number of lantibiotics have been shown to interfere with peptidoglycan biosynthesis by binding to lipid II, which intriguingly all act differently on lipid II, where different structures of these compounds are increasingly being focused to explain the sophisticated mode of actions directed by diverse structures of lantibiotics.

The prototypic type-A lantibiotic, nisin is an elongated amphipathic screw-shaped structure in solution, having a net positive charge. Initially, their bactericidal action was believed to predominantly involve the formation of short-lived pores in bacterial cell membranes (as mentioned before). More recently, unique mechanism of actions has been shown to be exerted by nisin that renders it highly potent with minimal concentrations against many Gram-positive bacteria in nanomolar concentration (5, 42). Many ambiguities have become clarified on mode of actions of lantibiotics after the report of Breukink et al. (5), suggesting that nisin interacts in a highly specific manner with lipid II. Dissimilar sensitivities of a lipid II targeting lantibiotic to different indicator strains may be due to the presence of different lipid II contents among various microorganisms (e.g., E. coli, $2 \times 10^{3}$ molecules per cell; Micrococcus lysodeikticus, $10^{5}$ molecules per cell) $(60,61)$. Many studies have subsequently proved that this inhibition is caused by binding to the lipid-associated peptidoglycan precursors lipid I and mostly to lipid II $(62,63)$. However, structural information on the interaction of lantibiotics with the cell wall precursor so far is restricted to lipid II. Nuclear magnetic resonance (NMR) data reveal that the pyrophosphate moiety of lipid II interacts with six hydrogen bonds to backbone amides of rings $\mathrm{A}$ and $\mathrm{B}$ of nisin (59).
Bonev et al. (64) reported that nisin can also bind with bactoprenol pyrophosphate; however, the affinity is considerably lower than that for the complete lipid II molecule, indicating that for high-affinity binding of nisin, additional interactions must take place, presumably between the $N$-acetylmuramyl moieties, whereas the pentapeptide side chain and the isoprenoid moiety would not be involved. Evidence for the interaction of the lantibiotics with lipid I stems mainly from the observation that lipid II biosynthesis is strongly blocked, but the structural analysis of a lantibiotic-lipid I complex has not yet been reported. The A and B ring system of nisin, which has been shown to be responsible for binding with lipid II, in particular the pyrophosphate moiety, is conserved in nisin, subtilin, epidermin, gallidermin, plantaricin $C$ and a number of structural variants, such as mutacin 1140 , mutacin $\mathrm{I}$, ericin $\mathrm{A}$, and streptin also show the similar binding motif. Bonelli et al. (43) showed that gallidermin/epidermin has higher affinity to lipid II compared to nisin and suggested that the structural element could be lysine at position 4 (isoleucine in nisin) that may provide an additional positive charge to enhance binding to the pyrophosphate moiety. Mersacidin, actagardin and cinnamycin are globular type-B lantibiotics and also bind to lipid II, but have no structural similarity with nisin and epidermin (Fig. 3). They act through disruption of enzyme function of cell wall biosynthesis by the formation of complex with lipid II $(65,66)$. Specifically, these compounds block the transglycosylation step of peptidoglycan biosynthesis (65). It is important to note that mersacidin does not form pores upon binding to lipid II that would be the reason for moderate MIC values. However, the compound is very effective in vivo against staphylococcal infections $(67,68)$, including MRSAand vancomycin-resistant enterococci (65). Mersacidin adopts a different structure with lipid II in dodecylphosphocholine micelles than that is observed in the absence of lipid II (66). In vitro peptidoglycan synthesis assays suggested that epidermin and nisin accumulated lipid I indicating that they could also inhibit the conversion of lipid I into lipid II (69).

\section{STRUCTURAL VARIANTS TO STUDY MODE OF ACTIONS}

Mutants and fragments generated by site-directed mutagenesis, chemical and enzymatic digestion from many works show enormous information for the modes of action of lantibiotics. Removal of Dha33 and Lys34 from the C-terminus of nisin does not affect antibacterial activity (70). Introduction of an additional positive charge by Val32Lys variant shows relatively little effect, whereas a negative charge (Val32Glu) provides about 4-fold decreased activity against some indicator strains (42). 
Epilancin K7 shares a very similar C-terminus double-ring system with nisin, which also does not show interaction with lipid II (69) that further supports the relatively unimportant role of C-terminus of these lantibiotics in biological activity. However, many studies strongly suggested that the N-terminus of nisin is essential for binding. For example, nisin fragment, which has no activity but shows antagonistic activity against nisin's bactericidal activity (35), indicating that the fragment competes for the same binding site to that of nisin. Complete proteolytic deletion of the $\mathrm{D}$ and $\mathrm{E}$ rings of nisin leads to a 100-fold decrease of activity (35), whereas chemical disruption of Dha5 that opens the A ring results in more than 500-fold reduction of antibacterial activity (70).

The lipid II variant containing shorter prenyl tail (3 from 11 isoprene units) can form complex with nisin and the length of this isoprene tail does not affect pore-forming activity (56). Intermolecular hydrogen bonds between the amides of Dhb2, Ala3, Ile4, Dha5, and Abu8 on nisin and the oxygens of the pyrophosphate group of lipid II hold the pyrophosphate moiety of lipid II within the cavity. Additionally, MurNAc and the first isoprene unit put together the binding site for the recognition of nisin. Replacement of Lan in the A ring of nisin to a MeLan resulted in 50-fold reduced affinity of the peptide to lipid II (42), and it is now well established that chemical opening of the A ring eventually causes complete loss of activity (70). The reasons for binding of nisin and epidermin to both lipid I and lipid II have been revealed by the NMR structure (69) in which both the peptide share the recognition motif. The explanation for the activity of nisin against vancomycin-resistant bacteria is that, nisin does not make contact with vancomycin' binding site (L-LysD-Ala-D-Ala moiety of pentapeptide) on lipid IILacticin 3147 (Fig. 3).This is an excellent example of the two-peptides lantibiotics for representing the exceptional antibiotic efficiency that are achieved when two killing mechanisms combine, though its high activities in the nanomolar concentration range are to some extent strain or species specific. Wiedemann et al. (71) reported that lacticin 3147 peptides (lntA1 and lnA2) have very strong synergistic effect against Lactococcus lactis, but remarkably lower against Micrococcus flavus. However, there is no satisfactory explanation for the fact that the A1 peptide and mersacidin are almost equally effective against the lactococcal strain, but differ by a factor of 30 against the Micrococcus. The activity of lacticin 3147 involves the binding of ltnA1 peptide to lipid II. Both activities (pore formation and inhibition of cell wall biosynthesis) require the presence of the two peptides whose intermolecular interactions appear to be stabilized by lipid II (71).

\section{INHIBITION OF SPORE GERMINATIONS}

Most studies are mainly inclined to the antibacterial activity determined against vegetative cells. Nisin, subtilin and sublancin inhibit the spores' outgrowths from Bacillus and Clostridium species $(72,73)$. This activity has been proposed to cause from covalent modification of a target on the spore coat by nucleophilic attack on Dha5 for nisin and subtilin (74). The reactive thiol groups on the exterior of spores from Bacillus cereus react with compounds such as $S$ nitrosothiols and iodoactetate, and nisin interferes with the modification of these sulfhydryl groups (75), suggesting that the target of nisin for inhibition of spore germination are these reactive thiol groups (76). However, covalent mechanism has not yet been established. Replacement of Dha5 by Ala via sitedirected mutagenesis of both subtilin $(74,77)$ and nisin (35) abolished the inhibition of spore germination indicating it as their putative site of attack. Ironically, the mutants showed no effect on the antibacterial activity of subtilin and nisin against vegetative cells of Bacillus cereus, and $L$. lactis and $M$. luteus, respectively. The above studies clearly suggest that the inhibition of spore germination is a different activity of these lantibiotics. Therefore, inhibition of spore outgrowth is the third distinct biological activity of lantibiotics with a different structure-function relationship.

\section{OTHER BIOLOGICAL FUNCTIONS AND MORE ARE YET TO BE DONE}

Many lantibiotics have their interesting biological activities rather functioning only as an antibacterial agent. The SapB (Fig. 3) peptide produced by Streptomyces coelicolor works as a morphogenic peptide (68). Another example is the novel lantibiotic sublancin (Fig. 3), which has lipid II-independent mode of actions, such as the induction of autolysis of staphylococci (19). Cinnamycin (Fig. 3) and duramycins strongly inhibit the phospholipase A2 by sequestering its phosphatidylethanolamine (PE) (7880 ), in addition to their bactericidal and hemolytic activities. Choung et al. (81) reported that cinnamycin induces transbilayer lipid movement, seemingly in a PE-dependent fashion (82). Nisin and Pep5 also induce autolysis of certain staphylococcal strains primarily by breaking down the cell wall at the septa of dividing cells additionally to their usual mode of actions. The associations of the positively charged lantibiotics with the negatively charged teichoic and of actiolipoteichoic acids displace and activate $N$-acetyl-L-alanine amidase and $N$-acetylglucosaminidase enzymes that are generally associated with the teichoic acids $(83,84)$. Not all lantibiotics bind to lipid I or lipid II to exert their mode of actions. In most cases, the molecules involved or the particular reasons that are attributed to the activity are yet to be identified. 
Pep5 and epilancin K7 have specifically been shown not to bind lipid I or lipid II (85) but these lantibiotics still have activities against some bacteria that are far greater than those of other pore-forming lantibiotics. High activity of Pep5 in nanomolar concentrations against Staphylococcus simulans and $S$. carnosus signify that it employs a different high-affinity receptor or docking molecule for its potent biological activity $(69,85)$. Therefore, as it is now working in many laboratories to unravel the mode of actions of many lantibiotics, there will hopefully be a wide varieties of lantibiotics with new era of amazing structure-based antibacterial activities.

\section{APPLICATIONS AND FUTURE POTENTIAL}

It is already mentioned that nisin has a widespread application as a food preservative in almost 50 countries for more than 40 years. Nisin is added to the positive list of food additive by the European Union and has also been approved by the Food and Drug Administration $(3,4)$. Since nisin is active against a wide range of bacteria, it is suitable for a number of different applications. Nisin, mutacin, mersacidin etc., are in preclinical stage to be used in medical applications (86). The most significant application of lantibiotics may be in the treatment of antibioticresistant pathogens. Ryan et al. (87) have gathered information about the potential biomedical applications of lantibiotics to be ventured in clinical and veterinary therapies. Nisin is effective against bacterial mastitis, oral hygiene, enterococcal infections, peptic ulcer treatment, treatment of enterocolitis, etc.; mersacidin and actagardine show remarkable activity against Staphylococcus aureus including MRSA, bacterial mastitis, oral hygiene, acne, etc.; gallidermin and epidermin are effective against acne, eczema, follicultis, impetigo, and can also be used for personal care products; mutacin 1140 can prevent dental caries; lacticin 3147 can prevent bacterial mastitis, MRSA and enterococcal infections, oral hygiene, acne; cinnamycin may be used for inflamation viral infection treatment and blood pressure regulation; and duramycin and ancovenin can be used for inflamation and blood pressure regulation, resepectively.

Since there is a recent threat of using spores of Bacillus anthracis in bioterrorism, the inhibitory activities of lantibiotics, such as, subtilin $(74,77)$ and nisin (35) against spore germination would have interesting and potential future applications.

\section{REFERENCES}

1. Weigel, L. M., et al. 2003. Genetic analysis of a high-level vancomycin resistant isolate of Staphylococcus aureus. Science 302:1569-1571.
2. WHO (World Health Organization). 2000. Press release, WHO/41. http://www.who.int 2000.

3. Delves-Broughton, J. 1990. Nisin and its uses as a food preservative. Food Technol. 44:100-117.

4. Delves-Broughton, J., P. Blackburn, R. J. Evans, and J. Hugenholtz. 1990. Applications of the bacteriocin, nisin. Antonie van Leeuwenhoek 69:193-202.

5. Breukink, E., et al. 1999. Use of the cell wall precursor lipid II by a pore-forming peptide antibiotic. Science 286:2361-2364.

6. Gross, E, and J. L. Morell. 1971. The structure of nisin. J. Am. Chem. Soc. 93:4634-4635.

7. Schnell, N., et al. 1988. Prepeptide sequence of epidermin, a ribosomally synthesized antibiotic with four sulphide-rings. Nature 333:276-278.

8. Mootz, H. D., and M. A. Marahiel. 1997. Biosynthetic systems for nonribosomal peptide antibiotic assembly. Curr. Opin. Chem. Biol. 1:543-551.

9. Nissen-Meyer, J., and I. F. Nes. 1997. Ribosomally synthesized antimicrobial peptides: their function, biogenesis, and mechanism of action. Arch. Microbiol. 167:67-77.

10. Tagg, J. R., A. S. Dajani, and L. W. Wannamaker. 1976. Bacteriocins of gram-positive bacteria. Bacteriol. Rev. 40:722-756.

11. De Vos, W. M., O. P. Kuipers, J. R. van der Meer, and R. J. Siezen. 1995. Maturation pathway of nisin and other lantibiotics: posttranslationally modified antimicrobial peptides exported by gram-positive bacteria. Mol. Microbiol. 17:427-437.

12. Sahl, H. G., R. W. Jack, and G. Bierbaum. 1995. Biosynthesis and biological activities of lantibiotics with unique post-translational modifications. Eur. J. Biochem. 230:827-853.

13. Jung, G. 1991. Lantibiotics - ribosomally synthesized biologically active polypeptides containing sulfide bridges and $\alpha, \beta$-didehydroamino acids. Ang. Chem. Intl. Ed. Engl. 30:1051-1068.

14. Van der Meer, J. R ., et al. 1994. Influence of amino acid substitutions in the nisin leader peptide on biosynthesis and secretion of nisin by Lactococcus lactis. J. Biol. Chem. 269:3555-3562.

15. Xie, L ., et al. 2004. Lacticin 481: In vitro reconstitution of lantibiotic synthetase activity. Science 303:679-681.

16. Majer, F., D. G. Schmid, K. Altena, G. Bierbaum, and T. Kupke. 2002. The flavoprotein MrsD catalyzes the oxidative decarboxylation reaction involved in formation of the peptidoglycan biosynthesis inhibitor mersacidin. J. Bacteriol. 184:1234-1243.

17. Schmid, D. G., F. Majer, T. Kupke, and G. Jung. 2002. Electrospray ionization fourier transform ion cyclotron resonance mass spectrometry to reveal the substrate specificity of the peptidyl-cysteine decarboxylase EpiD. Rapid. Commun. Mass Spectrom. 16:1779-1784.

18. Kuipers, A., et al. 2004. NisT, the transporter of the lantibiotic nisin, can transport fully modified, dehydrated and unmodified prenisin and fusions of the leader peptide with non-lantibiotic peptides. J. Biol. Chem. 279:22176-22182.

19. Havarstein, L. S., D. B. Diep, and I. F. Nes. 1995. A family of bacteriocin ABC transporters carry out proteolytic processing of their substrates concomitant with export. Mol. Microbiol.16:229-240.

20. Heidrich, C., et al. 1998. Isolation, characterization, and heterologous expression of the novel lantibiotic epicidin 280 and analysis of its biosynthetic gene cluster. Appl. Environ. Microbiol. 64:3140-3146.

21. de Ruyter, P. G., et al. 1996. Functional analysis of promoters in the nisin gene cluster of Lactococcus lactis. J. Bacteriol. 178:3434-3439.

22. Jung, G. 1991. Lantibiotics: a survey, p 1-34. In G. Jung, and H. G. Sahl (ed.), Nisin and novel lantibiotics. ESCOM, Leiden.

23. Nagao, J., et al. 2006. Lantibiotics: Insight and foresight for new paradigm. J. Biosci. Bioeng. 102:139-149.

24. Gilmore, M. S., et al. 1994. Genetic structure of the Enterococcus faecalis plasmid pAD1-encoded cytolytic toxin system and its relationship to lantibiotic determinants. J. Bacteriol. 176:7335-7344.

25. Pag, U., and H. G. Sahl. 2002. Multiple activities in lantibiotics--models for the design of novel antibiotics? Curr. Pharm. 8:815-833.

26. Mulders, J. W., et al. 1991. Identification and characterization of the lantibiotic nisin Z, a natural nisin variant. Eur. J. Biochem. 201:581-584.

27. Zendo, T., et al. 2003. Identification of the lantibiotic nisin Q, a new natural nisin variant produced by Lactococcus lactis 61-14 isolated from a river in Japan. Biosci. Biotechnol. Biochem. 67:1616-1619.

28. Morency, H., et al. 2001. Comparison of the activity spectra against pathogens of bacterial strains producing a mutacin or a lantibiotic. Can. J. Microbiol. 47:322-331.

29. Kellner, R., et al. 1988. Gallidermin: a new lanthionine-containing polypeptide antibiotic. Eur. J. Biochem. 177:53-59.

30. Dufour, A., T. Hindre, D. Haras, and J. P. L. Pennec. 2006. The biology of lantibiotics from the lacticn 481 group is coming of age FEMS Microbiol. Rev. (In press.) 
31. Kuipers, O. P., et al. 1992. Engineering dehydrated amino acid residues in the antimicrobial peptide nisin. J. Biol. Chem. 267:2434024346.

32. Kuipers, O. P., et al. 1996. Protein engineering of lantibiotics. Antonie van Leeuwenhoek 69:161-169.

33. Chen, P., et al. 1998. Structure-activity study of the lantibiotic mutacin II from Streptococcus mutans T8 by a gene replacement strategy. Appl. Environ. Microbiol. 64:2335-2340.

34. Bierbaum, G.., et al. 1996. Engineering of novel thioether bridge and role of modified residues in the lantibioitc Pep5. Appl. Environ. Microbial. 62:385-392.

35. Chan, W. C., et al. 1996. Structure-activity relationships in the peptide antibiotic nisin: role of dehydroalanine 5. Appl. Environ. Microbiol. 62:2966-2969.

36. Dodd, H. M., N. Horn, and M. J. Gasson. 1995. A cassette vector for protein engineering the lantibiotic nisin. Gene. 162:163-164.

37. Szekat, C., R. W. Jack, D, Skutlarek, H. Farber, and G. Bierbaum. 2003. Construction of an expression system for site-directed mutagenesis of the lantibiotic mersacidin. Appl. Environ. Microbiol. 69:3777-3783.

38. Szekat, C., et al. 2003. Construction of an expression system for sitedirected mutagenesis of the lantibiotic mersacidin. Appl. Environ. Microbiol. 69:3777-3783.

39. Kraaij, C. V., et al. 1997. Influence of charge differences in the Cterminal part of nisin on antimicrobial activity and signaling capacity. Eur. J. Biochem. 247:114-120.

40. Yuan, J., et al. 2004. Site-directed mutagenesis of the hinge region of nisin $\mathrm{Z}$ and properties of nisin $\mathrm{Z}$ mutants. Appl. Microbial Biotechnol. 64:806-815

41. Rollema, H. S., et al. 1995. Improvement of solubility of the antimicrobial peptide nisin by protein engineering. Appl. Environ. Microbial. 61:2873-2978

42. Wiedemann, I., et al. 2001. Specific binding of nisin to the peptidoglycan precursor lipid II combines pore formation and inhibition of cell wall biosynthesis for potent antibiotic activity. J. Biol. Chem. 276:1772-1779.

43. Bonelli, R. R., T. Schneider, H. G. Sahl, and I. Wiedemann. 2006 Insights into in vivo activities of lantibiotics from gallidermin and epidermin mode-of-action studies. Ant. Agents Chem. 50:1449-1457.

44. Giffard, C. J., et al. 1997. Structure-function relations of variant and fragments of nisin studied with model membrane systems. Biochemistry 36:3802-3810.

45. Breukink, E., et al. 1997. The C-terminal region of nisin is responsible for the initial interaction of nisin with the target membrane. Biochemistry 36:6968-6976.

46. Moll, G. N., et al. 1997. Role of transmembrane $\mathrm{pH}$ gradient and membrane binding in nisin pore formation. J. Bacteriol. 179:135-140.

47. Van Kraaij, C., et al. 1998. Pore formation by nisin involves translocation of its C-terminal part across the membrane. Biochemistry 37: 16033-16040.

48. Demel, R. A., et al. 1996. Nisin Z, mutant nisin Z and lacticin 481 interactions with anionic lipids correlate with antimicrobial activity. A monolayer study. Eur. J. Biochem. 235:267-274.

49. Sahl, H. G. 1991. Pore formation in bacterial membranes by cationic lantibiotics, p 347-358. In G. Jung, and H. G. Sahl, (ed.), Nisin and novel lantibiotics. ESCOM, Leiden.

50. Kordel, M., R. Benz, and H. G. Sahl. 1988. Mode of action of the staphylococcin like peptide Pep 5: voltage-dependent depolarization of bacterial and artificial membranes. J. Bacteriol. 170:84-88.

51. Sahl, H. G., M. Kordel, and R. Benz. 1987. Voltage-dependent depolarization of bacterial membranes and artificial lipid bilayers by the peptide antibiotic nisin. Arch. Microbiol. 149:120-124.

52. Benz, R., G. Jung, and H. G. Sahl. 1991. Mechanism of channelformation by lantibiotics in black lipid membranes, p 359-372. In G. Jung, and H. G. Sahl, (ed.), Nisin and novel lantibiotics. ESCOM, Leiden.

53. Gao, F. H., T. Abee, and W. N. Konings. 1991. Mechanism of action of the peptide antibiotic nisin in liposomes and cytochrome c oxidasecontaining proteoliposomes. Appl. Environ. Microbiol. 57:2164-2170.

54. Driessen, A. J., et al. 1995. Mechanistic studies of lantibiotic-induced permeabilization of phospholipid vesicles. Biochemistry 34:1606-1614.

55. Chikindas, M. L., et al. 1995. Mutacin II, a bactericidal antibiotic from Streptococcus mutans. Antimicrob. Agents Chemother. 39:265660.

56. Breukink, E., et al. 2003. Lipid II is an intrinsic component of the pore induced by nisin in bacterial membranes. J. Biol. Chem. 278: 1989819903.

57. Van Heusden, H. E., B. de Kruijff, and E. Breukink. 2002. Lipid II induces a transmembrane orientation of the pore-forming peptide lantibiotic nisin. Biochemistry 41:12171-12178.

58. Matsuzaki, K. 1999. Why and how are peptide-lipid interactions utilized for self-defense? Magainins and tachyplesins as archetypes. Biochim. Biophys. Acta. 1462:1-10.
59. Hsu, S. T. D., et al. 2004. The nisin-lipid II complex reveals a pyrophosphate cage that provides a blueprint for novel antibiotics. Nat. Struct. Mol. Biol. 11:963-967.

60. Storm, D. R., and J. Strominger. 1974. Binding of bacitracin to cells and protoplasts of Micrococcus lysodeikticus. J. Biol. Chem. 249:1823-1827.

61. Van Heijenoort, Y., et al. 1992. Membrane intermediates in the peptidoglycan metabolism of Escherichia coli: possible roles of PBP 1b and PBP 3. J. Bacteriol. 174:3549-3557.

62. Chatterjee, C., M. Paul, L. Xie, and W. A. van der Donk. 2005 Biosynthesis and mode of action of lantibiotics. Chem. Rev. 105:633-684.

63. Reisinger, P. H. Seidel, H. Tschesche, and W. P. Hammes. 1980. The effect of nisin on murein synthesis. Arch. Microbiol. 127:187-193.

64. Bonev, B. B.,et al. 2004. Targeting extracellular pyrophosphates underpins the high selectivity of nisin. FASEB J. 18:1862-1869.

65. Ottenwälder, B., et al. 1995. Isolation and characterization of genetically engineered gallidermin and epidermin analogs. Appl. Environ. Microbiol. 61:3894-3903.

66. Hsu, S. T. D., et al. 2003. NMR study of mersacidin and lipid II interaction in dodecylphosphocholine micelles. Conformational changes are a key to antimicrobial activity. J. Biol. Chem. 278:13110-13117.

67. Chatterjee, S., et al. 1992. Mersacidin, a new antibiotic from Bacillus: fermentation, isolation, purification, and chemical characterization. J. Antibiot. (Tokyo). 45:832-838.

68. Limbert, M., et al. 1991. Chemotherapeutic properties of mersacidin in vitro and in vivo, p. 448-456. In G. Jung, and H. G. Sahl (ed.) Nisin and novel lantibiotics. ESCOM, Leiden.

69. Brötz, H., et al. 1998. Role of lipid-bound peptidoglycan precursors in the formation of pores by nisin, epidermin and other lantibiotics. Mol. Microbiol. 30:317-327.

70. Chan, W. C., B. W. Bycroft, L. Y. Lian, and G. C. Roberts. 1989 Isolation and characterisation of two degradation products derived from the peptide antibiotic nisin. FEBS Lett. 252:29-36.

71. Wiedemann, I., et al. 2006. The mode of action of the lantibiotic lacticin 3147 - a complex mechanism involving specific interaction of two peptides and the cell wall precursor lipid II. Mol. Microbiol. 61:285-297.

72. Hurst, A. 1981. Nisin. Adv. Appl. Microbiol. 27:85-123.

73. Paik, S. H., A. Chakicherla, and J. N. Hansen. 1998. Identification and characterization of the structural and transporter genes for, and the chemical and biological properties of, sublancin 168, a novel lantibiotic produced by Bacillus subtilis 168. J. Biol. Chem. 273:23134-23142.

74. Liu, W., and J. N. Hansen. 1993. The antimicrobial effect of a structura variant of subtilin against outgrowing Bacillus cereus $\mathrm{T}$ spores and vegetative cells occurs by different mechanisms. Appl. Environ. Microbiol. 59: 648-651.

75. Morris, S. L., and J. N. Hansen. 1981. Inhibition of Bacillus cereus spore outgrowth by covalent modification of a sulfhydryl group by nitrosothiol and iodoacetate. J. Bacteriol. 148:465-471.

76. Morris, S. L., R. C. Walsh, and J. N. Hansen. 1984. Identification and characterization of some bacterial membrane sulfhydryl groups which are targets of bacteriostatic and antibiotic action. J. Biol. Chem. 259:13590 13594.

77. Liu, W., and J. N. Hansen. 1992. Enhancement of the chemical and antimicrobial properties of subtilin by site-directed mutagenesis. J. Biol. Chem. 267:25078-085.

78. Fredenhagen, A., et al. 1991. Duramycin B and C, two new lanthioninecontaining antibiotics as inhibitors of phospholipase A2, and structural revision of duramycin and cinamycin, p 131-140. In G. Jung, and H. G. Sahl (ed.), Nisin and novel lantibiotics. ESCOM, Leiden.

79. Machaidze, G. and J. Seelig. 2003. Specific binding of cinnamycin (Ro 09-0198) to phosphatidylethanolamine. Comparison between micellar and membrane environments. Biochemistry 42:12570-12576.

80. Märki, F., E. Hanni, A. Fredenhagen, and J. van Oostrum. 1991. Mode of action of the lanthionine-containing peptide antibiotics duramycin, duramycin B and C, and cinnamycin as indirect inhibitors of phospholipase A2. J. Biochem. Pharmacol. 42:2027-2035.

81. Choung, S., et al. 1988. Hemolytic activity of a cyclic peptide Ro09-0198 isolated from Streptoverticillium. Biochim. Biophys. Acta. 940:171-179.

82. Makino, A., et al. 2003. Cinnamycin (Ro 09-0198) promotes cell binding and toxicity by inducing transbilayer lipid movement. J. Biol. Chem. 278:3204-3209.

83. Bierbaum, G., and H. G. Sahl. 1985. Induction of autolysis of staphylococci by the basic peptide antibiotics Pep 5 and nisin and their influence on the activity of autolytic enzymes. Arch. Microbiol. 141:249 254.

84. Bierbaum, G., and H. G. Sahl. 1987. Autolytic system of Staphylococcus simulans 22: influence of cationic peptides on activity of $\mathrm{N}$ acetylmuramoyl-L-alanine amidase. J. Bacteriol. 169:5452-5458.

85. Pag, U., and H. G. Sahl. 2002. Multiple activities in lantibiotics--models for the design of novel antibiotics? Curr. Pharm. 8:815-833.

86. Breukink, E., and B. de Kruijff. 2006. Lipid II as a target for antibiotics. Nat. Rev. Drug Discov. 5:321-32.

87. Ryan, M. P., C. Hill, and R. P. Ross. 2002. Exploitation of lantibiotic peptides for food and medical uses, p. 193-242. In Dutton, C. J., M. A. Haxell, H. A. I. McArthur, and R. G. Wax (ed.), Peptide antibiotics discovery, mode of action and applications. Marcel Dekker, New York. 